\title{
EDITORIAL
}

\section{Un nuevo reto para Salud Pública de México}

D urante estos últimos tres años Salud Pública de México ha emprendido diversas actividades para fortalecer su contenido científico y ampliar su visibilidad internacional. La meta es llegar a ser el mejor foro de difusión de la investigación en salud pública de la región latinoamericana.

El balance de estos últimos años es bastante alentador: se han dado pasos importantes para fortalecer el carácter científico de la revista y, al mismo tiempo, se han identificado áreas que requieren de mayor atención y desarrollo. Este es un momento propicio para compartirlo con nuestros lectores y con autores que han contribuido con sus valiosos manuscritos, así como con tareas de revisión y dictamen de artículos que se someten a la revista.

Entre los atributos que comúnmente destacan en las revistas con mayor reconocimiento internacional son la regularidad y la puntualidad de publicación, el contenido científico, el registro de la revista en índices internacionales ampliamente difundidos en la comunidad científica, la composición del comité editorial y el factor de impacto de citaciones de sus artículos. ${ }^{1,2}$

Salud Pública de México, en sus 40 años de ininterrumpida publicación, ha hecho esfuerzos por mantener una puntualidad mayor a la del promedio de las revistas latinoamericanas, lo cual hace que destaque entre las escasas publicaciones en español que están registradas en índices internacionales. Es una de las diez revistas biomédicas mexicanas incorporadas al Index Medicus y una de las cinco del área de la salud registradas en el Indice de Revistas Mexicanas de Investigación Científica del CONACyT. Además, esta publicación forma parte de la base de datos de Excerpta Medica y del Current Contents. Esto, sin duda, contribuye a ampliar su proyección como un medio de difusión destacado en el campo de la salud pública latinoamericana.
Hay otros atributos que esta revista ha ido fortaleciendo y que conviene mencionar. En estos últimos años, por ejemplo, se observa que el mayor número de manuscritos publicados son investigaciones originales $(68 \%)$, escritas por investigadores con estudios de posgrado (62\%); asimismo, $76 \%$ de los autores son externos al Instituto Nacional de Salud Pública. ${ }^{3}$ Sin embargo, las contribuciones de investigadores extranjeros continúan siendo insuficientes, aun cuando desde 1993 Salud Pública de México publica en los idiomas español e inglés. El proceso de dictaminación de los trabajos hasta su publicación se realiza en un periodo promedio de 7.5 meses, lo cual la hace comparable a un gran número de revistas con reconocimiento internacional.

Uno de los aspectos que intervienen en la calidad del contenido de la revista es el proceso de revisión por pares que se utiliza en la dictaminación de los trabajos. En efecto, el rigor que se aplica a este proceso y el apego a los lineamientos editoriales que se establecen en el ámbito internacional para las publicaciones científicas se reflejan en la tasa de rechazo de los trabajos que se someten a dictamen. Asimismo, la organización y la fluidez en los procesos de recepción, revisión y dictaminación influyen en el tiempo que demora la revista en publicar los manuscritos y, por tanto, en la oportunidad con la que los resultados de las investigaciones llegan al lector.

Para agilizar el proceso de dictaminación se creó el Consejo de Editores de Sección con la finalidad de realizar una primera revisión y selección cuidadosa de los artículos y de los dictaminadores. Sin duda, los nuevos procedimientos han ayudado a elevar la calidad de la revisión y, por lo tanto, a incrementar el índice de rechazo. Actualmente el porcentaje de artículos rechazados es de $63 \% \mathrm{y}$, aunque es menor al de otras revistas internacionales, se ha ido incrementando durante 
los últimos años; actualmente es uno de los más altos entre las revistas biomédicas mexicanas.

Por otro lado, la composición del Comité Editorial y la participación de sus miembros en el quehacer editorial son recursos importantes para mantener la calidad y la credibilidad de una revista. Para el Index Medicus, por ejemplo, lo anterior representa un requisito para considerar las publicaciones que pasan a formar parte de este índice. En la actualidad el Comité Editorial de Salud Pública de México cuenta con 33 miembros de gran prestigio en la comunidad científica; $37 \%$ de ellos son del extranjero; $44 \%$, de otras instituciones mexicanas, y 19\%, del Instituto Nacional de Salud Pública.

Sin duda, Salud Pública de México ha ido mejorando paulatinamente. Sin embargo, aún quedan muchos desafíos por vencer, especialmente en estos años de transición, cuando no sólo las condiciones de la salud de las poblaciones muestran nuevas modalidades, sino también cuando la propia publicación científica se ve cada vez más involucrada en una ola de grandes transformaciones tecnológicas.

Son muchas las interrogantes que surgen frente a un futuro caracterizado por incertidumbres: ¿cómo ampliar la presencia de esta revista en la comunidad internacional?, ¿cómo incrementar su índice de impacto?, ¿cuál debe ser el idioma de publicación?, ¿cómo aumentar la oferta de artículos para publicar?, ¿debe publicarse en formato electrónico o continuar con el formato actual? En fin, éstas han sido algunas de las preguntas que surgen regularmente y que el staff de la revista consideró conveniente plantear a su Comité Editorial. Había pasado tiempo sin que sus miembros tuvieran la oportunidad de compartir los avances de la revista y los retos que se avecinan.

En efecto, el 9 de marzo de este año se llevó a cabo una reunión de trabajo con los miembros del Comité Editorial a la que asistieron académicos mexicanos y extranjeros. Se plantearon dos objetivos fundamentales: a) analizar las actuales condiciones de desarrollo de la revista y los aspectos que se deben fortalecer para asegurar una mayor calidad de contenido, más visibi- lidad y un mayor impacto internacional, y b) iniciar una mayor comunicación e intercambio con los miembros del Comité, con la finalidad de involucrarlos más en el nuevo curso que inicia Salud Pública de México.

Los resultados de esta reunión superaron las expectativas. Los asistentes demostraron un serio interés por contribuir con ideas novedosas para lograr los propósitos establecidos. Hubo consenso en considerar que Salud Pública de México es una buena publicación y que los aspectos anteriormente planteados son los que se deben fortalecer. Las sugerencias fueron numerosas y oportunas para ser aplicadas en el corto y mediano plazo. Resaltan algunos aspectos que nos han parecido de especial importancia, como son: la necesidad de hacer énfasis en su carácter científico y en su enfoque interdisciplinario; incrementar la presencia, dentro del Comité Editorial, de académicos provenientes de disciplinas afines y pertenecientes a la comunidad científica latinoamericana; crear secciones que estimulen el debate sobre temas de actualidad y de mayor relevancia en la salud pública; ampliar la distribución de la revista en Iberoamérica, y editar algunos números especiales en inglés para extender su presencia en otras regiones del mundo.

Tenemos confianza en que la mayor calidad de nuestra revista se traducirá, eventualmente, en mayor conocimiento y mejores políticas de salud en países en desarrollo.

Jaime Sepúlveda*

\section{Referencias}

1. Coloaianni LA. Selección de revistas en el Index Medicus. Memorias de la Primera Reunión de Editores de Revistas Biomédicas Mexicanas; 1994 marzo 4; México, D.F.

2. Awad R, Faba G, García C, Lezana MA, Loria A, 0 ropeza $C$ et al. La evaluación de las revistas biomédicas mexicanas. Revista del Instituto N acional de Enfermedades Respiratorias 1994; 7(3):230-235.

3. $O$ ropeza-A búndez C. Balance 1997. Salud Publica Mex 1998; 40:86-87.

* Director de Salud Pública de M éxico. 